\title{
On the use of artificial neural networks and micromechanical analysis for prediciting elastic properties of unidirectional composites
}

\author{
$\underline{\text { Ehsan Ghane }}^{1}$, Martin Fagerström $^{2}$, Mohsen Mirkhalaf ${ }^{1, *}$ \\ ${ }^{1}$ Department of Physics, University of Gothenburg, Gothenburg, Sweden \\ 2 Division of Material and Computational Mechanics, Department of Industrial and Materials Science, \\ Chalmers University of Technology, Gothenburg, Sweden \\ * mohsen.mirkhalaf@physics.gu.se
}

The composite design industry has a central demand to predict the elastic behavior of composites from their constituent properties and micromechanical information. In this case, the complex architecture of interlaced yarns in woven composites brings about challenges to accurately predict their mechanical behavior. Multiscale computational methods, often based on computational homogenization, have therefore been established to address the complexity in modeling woven composites. But for computational homogenization of woven composites, one needs to consider the microscale mechanical properties at every point inside a mesoscale unit cell. Based on the possible range of microstructural features, a plethora of research exists to generate random distributions of fibers in a microscopic representative volume element (RVE) and predict elastic properties using numerical methods, such as the finite element method [1,2]. But there is still a requirement to observe the whole possible microstructural design space based on any possible loading case and architecture in order to reach a generic model.

Recently, combining artificial neural network (ANN) and multiscale modeling has found stateof-the-art applications and opportunities. ANN is favorable at integrating multiscale data concerning correlations between different features. Following the work by Mentges et al. [3] on short fiber-reinforced composites, in this work, an ANN is used to replace the timeconsuming simulation of all 3D RVEs at the microscale to obtain elastic mesoscale properties. As such, the ANN model can be used on the mesoscale to compute the elastic, macroscopic properties of woven composites. The current study particularly aims to meet the high computational cost of simulating each design point through the training set using the minimum number of training data from the upper and lower limits of the design space. To design the training set, an automatic process is arranged to run Digimat-FE software to generate and analyze 3D unidirectional RVEs. Then, different feed-forward learning algorithms are employed to predict the test set through interpolation. Finally, a comprehensive study is done on the predicted (interpolary) results and the algorithmic operation.

\section{References}

[1] Melro AR, Camanho PP, Pinho ST. Generation of random distribution of fibres in long-fibre reinforced composites. Composite Science Technology 2008;68:2092-102. https://doi.org/10.1016/j.compscitech.2008.03.013.

[2] Tavares RP, Otero F, Baiges J, Turon A, Camanho PP. A dynamic spring element model for the prediction of longitudinal failure of polymer composites. Computer Material Science 2019;160:42-52. https://doi.org/10.1016/j.commatsci.2018.12.048.

[3] Mentges N, Dashtbozorg B, Mirkhalaf SM. A micromechanics-based artificial neural networks model for elastic properties of short fiber composites. Composite Part B Engineering 2021:108736. 